\title{
Numerical Study of Single-Hole and Multi-Holes Orifice Flow Parameters
}

\author{
M. Đurđević ${ }^{\dagger}$, M. Bukurov, S. Tašin and S. Bikić \\ Department of Energy and Process Engineering, Faculty of Technical Sciences, University of Novi Sad, Trg \\ Dositeja Obradovića 6, 21000 Novi Sad, Serbia
}

$\dagger$ Corresponding Author Email: markodj@uns.ac.rs

(Received February 12, 2020; accepted June 17, 2020)

\begin{abstract}
Importance of accurate fluid flow measurement in industry is crucial especially today with rising energy prices. There is no ideal measuring instrument due to numerous errors occurring during process of physical quantities measurement but also due to specific requirements certain instruments have like fluid type, installation requirements, measuring range etc. Each measuring instrument has its pros and cons represented in accuracy, repeatability, resolution, etc. Conventional single-hole orifice (SHO) flow meter is a very popular differential-pressure-based measuring instrument, but it has certain disadvantages that can be overcame by multi-holes orifice (MHO) flow meter. Having this in mind, the aim of this paper is to help gain more information about MHO flow meters. Both SHO and MHO gas (air) flow meters with same total orifice area and the pipe area ratio $\beta$ were numerically studied and compared using computational fluid dynamics (CFD). Simulation results of 16 different orifices with four different $\beta(0.5,0.55,0.6$ and 0.7$)$ were analysed through pressure drop and singular pressure loss coefficient. Standard $k-\varepsilon$ turbulence model was used as a turbulence model. Beside singular pressure loss coefficient, pressure recovery as well as axial velocity for both the SHO and MHO were reported. Results showed lower (better) singular pressure loss coefficient and pressure drop as well as quicker pressure recovery in favour of the MHO flow meters. Also, centreline axial velocity results were lower for MHO compared to corresponding SHO. CFD simulation results were verified by experimental results where air was used as a working fluid. The influence of geometrical and flow parameters on singular pressure loss coefficient was also reported and results showed that MHO hole distribution did not have significant influence on singular pressure loss coefficient.
\end{abstract}

Key words: CFD; Orifice flow meter; Multi-hole orifice flow meter; Pressure loss coefficient.

\section{NOMENCLATURE}

$A$ SHO opening area

$A_{1} \quad$ MHO central opening area

$A_{2} \quad$ MHO peripheral opening area

$A_{o} \quad$ total opening area (SHO and $\left.\mathrm{MHO}\right)$

$A_{p} \quad$ pipe area

$C_{\mu} \quad$ turbulent viscosity constant

$C_{d} \quad$ discharge coefficient

$D \quad$ internal pipe diameter

$d_{1} \quad$ SHO and MHO central hole diameter

$d_{2} \quad$ MHO peripheral hole diameter

$d_{\mathrm{c}} \quad$ MHO homocentric circle diameter

$e \quad$ thickness of the orifice

$E \quad$ thickness of the plate

$G_{k} \quad$ turbulent production $k \quad$ turbulence kinetic energy

$\dot{m}$ mass flow rate

$p \quad$ absolute pressure

$p_{1} \quad$ gauge pressure upstream of the orifice

$Q \quad$ volumetric flow rate

$R \quad$ universal gas constant

Re Reynolds number

$S \quad$ strain rate parameter

$T$ temperature

$Z \quad$ axial distance

Z compressibility factor

$Z_{n} \quad$ dimensionless axial distance

$\Delta p \quad$ pressure drop

$\mu \quad$ dynamic viscosity 


$\begin{array}{ll}\mu_{t} & \text { turbulent viscosity } \\ p_{\text {in }} & \text { upstream absolute pressure } \\ P_{n} & \text { dimensionless pressure } \\ P_{z} & \text { absolute pressure at axial distance } \\ \alpha & \text { angle of bevel } \\ \beta & \text { total orifice opening area and the pipe area } \\ & \text { ratio } \\ \varepsilon & \text { expansibility (expansion) factor }\end{array}$

\section{INTRODUCTION}

Many industries, especially energy industry is relaying on accurate flow measurements. Differential-pressure-based instruments are widely spread measuring devices with estimates that at least $40 \%$ of industrial flowmeters in use nowadays are differential-pressure-based where orifice flow meter is the most popular one (Reader-Harris, 2015). Installing an orifice plate inside a pipe carrying fluid, causes a pressure drop, making it possible to indirectly determine flow rate by measuring pressure difference upstream and downstream of the orifice. This procedure is described in standard EN ISO 5167-2:2012 where the flow rate is given by:

$\dot{m}=\frac{C_{d} \varepsilon}{\sqrt{1-\beta^{4}}} \frac{d^{2} \pi}{4} \sqrt{2 \rho \Delta p}$

The parameter $\beta$ is represented as ratio of square root of total orifice opening area and the pipe area.

$\beta=\sqrt{\frac{A_{o}}{A_{p}}}$

Orifice flow meters were topics of numerous experimental researches with different fluids and flow regimes. Teyssandier and Husain (1987) experimentally studied wall and orifice plate pressure gradients in air flows over a wide range of Reynolds numbers from 21000 to 160000 . Pressure loss coefficients for square-edged orifices with $\beta$ ratios of $0.2,0.3,0.57$ and 0.7 for Newtonian and non-Newtonian fluids in both laminar and turbulent flow regimes was experimentally determined by Ntamba Ntamba and Fester (2012). Over the years with growing application of computers in engineering, orifice flow meters became topic of numerous numerical studies. In last two decades CFD proved as a versatile tool for flow meters flow prediction (Singh et al. 2010). As a starting point for the simulation of fluid flow through the orifice plate, problems of flow simulation through the restricted pipe can be observed. In one of such papers Durst and Wang ( 1989) found a good match between the simulation results and the experimental measurements. In numerical simulations, the $k-\varepsilon$ turbulence model was used, while the velocity was measured using the laser Doppler anemometer (LDA). The pressure drop caused by the fluid flow through the pipe restriction was not presented. Erdal $\varepsilon \quad$ turbulence dissipation rate

$\xi \quad$ singular pressure loss coefficient

$\rho$ density

$\sigma_{k}, \sigma_{\varepsilon} \quad$ turbulent Schmidt number

$\tau$

$v$

shear stress

average velocity through the orifice opening area

$v_{a} \quad$ centreline axial velocity

and Andersson in their paper (Erdal and Andersson, 1997) presented the effects of the mesh, boundary conditions, the discretization schemes and the turbulence models on gas flow simulation through the pipe restriction. Good agreement between simulation results and experimental measurements authors achieved using standard $k-\varepsilon$ turbulence model. Despite the use of few different turbulence models it was not possible to predict the accurate pressure drop at the pipe restriction. Gan and Riffat (1997) studied pressure loss characteristics of square edged orifice and perforated plates in square ducts. They used CFD simulations to predict pressure distribution and (pressure) loss coefficient and to study effects of plate thickness. Ellman and Piche (1999) proposed a two-regime flow formula, which has a smooth transition between laminar and transition regimes for numerical simulations of fluid power circuits. Eiamsa-Ard et al. (2008) investigated the effects of $\beta$ (for $0.5,0.6$ and 0.8 ) on the flow field of single-hole orifice using both the standard $k-\varepsilon$ turbulence model and Reynolds stress model (RSM). Simulation results of both the standard $k-\varepsilon$ turbulence model and RMS are in good agreement with experimental measurements (Eiamsa-Ard et al., 2008). Drainy et al. (2009) in their numerical study of Zanker plate compared results of different turbulence models (standard k- $\varepsilon$, realizable k- $\varepsilon$, Reynolds Stress Model - RSM, large Eddy Simulation - LES, and Detached Eddy Simulation - DES) with pressure drop correlations of BS EN ISO 5167-2:2003 while also studying the effects of plate thickness and Reynolds number on flow characteristics of Zanker plate. Numerical methodology for predicting the calibration coefficients of the orifice meter using water as a fluid was presented by Oliveira et al. (2010). Arun et al. (2010) carried out CFD simulations to study effects of different $\beta(0.5,0.6$ and 0.8$)$ and pipe diameter for non-Newtonian fluid. Hollingshead et al. (2011) numerically studied discharge coefficients of different pressure differential devices (Venturi, standard orifice plate, V-cone and wedge flow meters) at low Reynolds numbers. Shah et al. (2012) presented a comprehensive numerical study of flow through a single-hole orifice meter. Authors validated CFD simulation results with published experimental data of Nail (1991) and Morrison et al. (1993) as well as with pressure drop and energy balance of their experimental data. Reader-Harris et al. (2012) simulated contamination of the orifice plate both by experiment and CFD simulations and good agreement between the two was obtained. 
Elsaey et al. (2014) numerically studied turbulent flow through fractal-shaped orifice with air as a working fluid. Shaaban (2014) studied orifice meter's energy consumption optimization using numerical simulations. Dong et al. (2018) studied measuring accuracy of the traditional stainless-steel orifice flowmeter and improved carbide orifice flowmeter by changing the entrance sharpness of the two orifices using CFD. Morrison et al. (1994) presented a slotted orifice flow meter as a replacement for conventional single hole orifice. Comparing experimental results of conventional single-hole orifice and slotted orifice flow meter proved that slotted orifice flow meter has much greater discharge coefficient and that its substantially less sensitive to upstream flow conditions. Barki and Ganesha (2014) compared single-hole orifice and multi-hole orifice using water as a fluid in their CFD simulations. Besides comparison of SHO and $\mathrm{MHO}$, authors studied the effects of multi-hole orifice plate hole distribution on flow rate, pressure drop, velocity and turbulence intensity. Numerical analysis showed better flow characteristics of MHO comparing to SHO. Singh and Tharakan (2015) numerically studied SHO and MHO flow meters over a wide range of Reynolds numbers using demineralized water as fluid. Comparing $\mathrm{SHO}$ and $\mathrm{MHO}$ flow meters results proved that MHO has better pressure recovery, greater discharge coefficient and lower downstream velocity.

In this paper authors present CFD simulation results of SHO and MHO flow meters singular pressure loss coefficient, flow patterns and pressure recovery. CFD simulation results were compared with experimental results and good agreement was obtained. All analyses in CFD simulations and experiments considered fluid (air) compressibility, where fluid density was defined as:

$$
\rho=\frac{p}{Z R T}
$$

To calculate discharge coefficient $C_{d}$ from Eq. (1) expansion factor $\varepsilon$ needs to be determined. Expansion factor $\varepsilon$ is determined experimentally, but for the MHO it wasn't determined, so singular pressure loss coefficient $\xi$ was introduced as a parameter for the analysis and comparison of SHO and MHO. Expressions for singular pressure loss coefficient are:

$$
\xi=\frac{\Delta p}{0.5 \rho v^{2}}
$$

and

$$
\xi=\frac{1-\beta^{4}}{\left(C_{d} \varepsilon\right)^{2}}
$$

The open literature contains no information on simulated MHO geometry for gaseous fluids. In total 16 orifices with four different $\beta(0.5,0.55,0.6$ and 0.7$)$ were studied using commercial CFD code Simcenter Star CCM+, Siemens. Input parameters $\beta$ ratio, homocentric circle diameter $d_{\mathrm{c}}$ (hole distribution) and Reynolds number were varied, while singular pressure loss coefficient $\xi$ and pressure recovery were analysed. The parameter $\beta$ and homocentric circle diameter $d_{\mathrm{c}}$ were parameters that were varied by changing orifice geometry, while Reynolds numbers were varied by changing air velocity. To analyse pressure recovery two nondimensional values were introduced, $P_{n}$ and $Z_{n}$ that are defined as:

$$
\begin{aligned}
& P_{n}=\frac{P_{z}}{P_{\text {in }}} \\
& Z_{n}=\frac{Z}{D}
\end{aligned}
$$

\section{ORIFICE AND MULTI-HOLE ORIFICE DESIGN}

Geometry of simulated MHO is similar to MHO designed by Singh and Tharakan (2015), who numerically studied MHO with same $\beta$ ratio, while demineralized water was used as a working fluid. Authors motivation for the current study was to see how these orifices will react in gaseous fluids. Out of 16 orifice plates used in this study, 4 were singlehole orifices (SHO) and 12 multi-hole orifices (MHO), with 4 different $\beta$ ratios $(0.5,0.55,0.6$ and $0.7)$ where for each $\beta$ one SHO and three MHO were designed. MHO were designed in the way having equal open area for flow as $\mathrm{SHO}$ with corresponding $\beta$. The MHO plates were designed with eight smaller circular openings evenly distributed on a homocentric circle in addition to the central opening. The details of the different multi-hole and single hole orifice plates used for the computations in this study are given in Table 1 and shown in Fig. 1 a).

In CFD model certain geometry approximations were made. Figure 1 shows approximated CFD

\begin{tabular}{|c|c|c|c|c|c|c|}
\hline$\beta[-]$ & $\begin{array}{c}D \\
{[\mathrm{~mm}]}\end{array}$ & Orifice & $\begin{array}{c}E \\
{[\mathrm{~mm}]}\end{array}$ & $\begin{array}{c}d_{1} \\
{[\mathrm{~mm}]}\end{array}$ & $\begin{array}{c}d_{2} \\
{[\mathrm{~mm}]}\end{array}$ & $\begin{array}{c}d_{\mathrm{c}} \\
{[\mathrm{mm}]}\end{array}$ \\
\hline \multirow{4}{*}{0.5} & \multirow{4}{*}{70.3} & SHO & 3.5 & 35.2 & - & - \\
\hline & & MHO1 & 3.5 & 18.0 & 10.7 & 40.0 \\
\hline & & MHO2 & 3.5 & 18.0 & 10.7 & 45.0 \\
\hline & & MHO3 & 3.5 & 18.0 & 10.7 & 52.0 \\
\hline \multirow{4}{*}{0.55} & \multirow{4}{*}{70.3} & SHO & 3.5 & 38.7 & - & - \\
\hline & & MHO1 & 3.5 & 21.0 & 11.5 & 43.0 \\
\hline & & MHO2 & 3.5 & 21.0 & 11.5 & 47.0 \\
\hline & & MHO3 & 3.5 & 21.0 & 11.5 & 52.0 \\
\hline \multirow{4}{*}{0.6} & \multirow{4}{*}{70.3} & SHO & 3.5 & 42.2 & - & - \\
\hline & & MHO1 & 3.5 & 23.0 & 12.5 & 42.0 \\
\hline & & MHO2 & 3.5 & 23.0 & 12.5 & 49.0 \\
\hline & & MHO3 & 3.5 & 23.0 & 12.5 & 54.0 \\
\hline \multirow{4}{*}{$\mid 0.7$} & \multirow{4}{*}{70.3} & SHO & 3.5 & 49.2 & - & - \\
\hline & & MHO1 & 3.5 & 28.0 & 14.3 & 48.5 \\
\hline & & MHO2 & 3.5 & 28.0 & 14.3 & 50.0 \\
\hline & & MHO3 & 3.5 & 28.0 & 14.3 & 53.0 \\
\hline
\end{tabular}
model geometry without bevel angle $\alpha=30^{\circ}$ (a) and geometry of experimentally tested orifices with bevel angle $\alpha=30^{\circ}$ (b).

Table 1 SHO and MHO orifice dimensions 

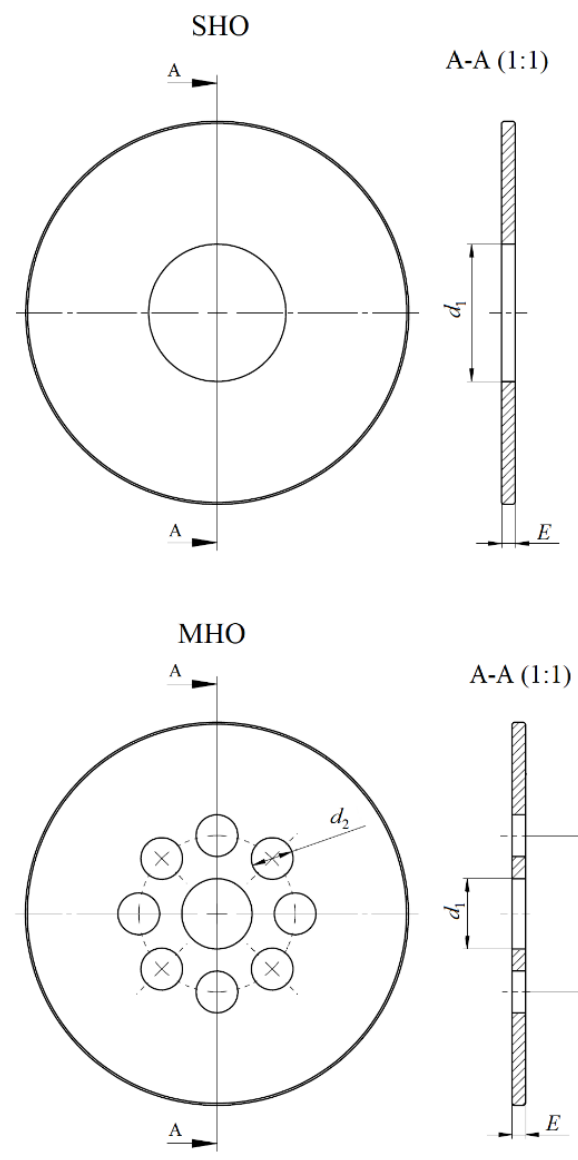

A-A (1:1)

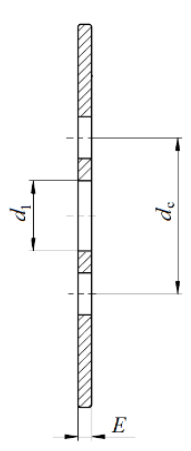

a) CFD orifice model geometry
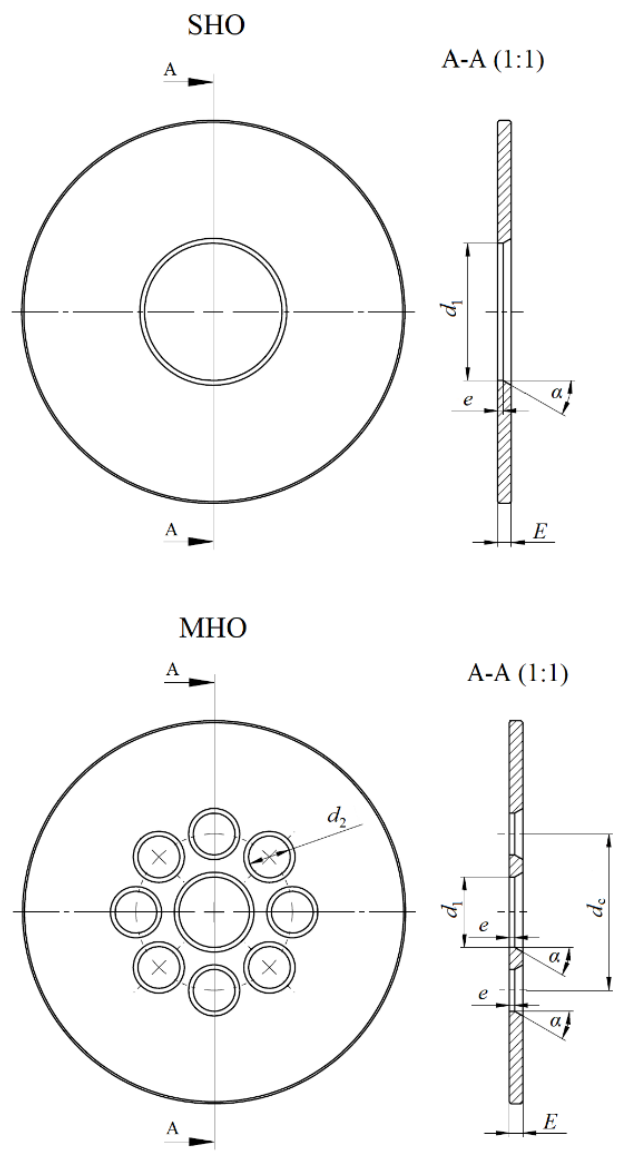

b) Tested orifice model geometry

Fig. 1. CFD and tested orifice geometry.

MHO plate works on the same principal as SHO causing pressure drop which is indirectly used to determine flow rate. Although orifice flow meters are widely spread measuring instruments, there is no available standard covering $\mathrm{MHO}$, but Eq. (1) can be used to determine the flow rate.

\section{CFD MODEL AND NUMERICAL PROCEDURE}

CFD simulations for total of 16 orifice pates were carried out under fully developed laminar and turbulent conditions in a $70.3 \mathrm{~mm}$ internal diameter and $3940.3 \mathrm{~mm}$ long pipe. Upstream section of 12 $D$ and downstream section of $44 D$ with orifice plate thickness of $3.5 \mathrm{~mm}$ were modelled (Fig. 2). To obtain the Reynolds numbers variation from 500 to 600000 , air mass flow rate was varied from $0.00051 \mathrm{~kg} / \mathrm{s}$ to $0.61415 \mathrm{~kg} / \mathrm{s}$.

\subsection{Mesh design}

Generating a high-quality grid is one of key issues in CFD simulations as it governs the stability and accuracy of the flow predictions. For the present study of SHO and MHO unstructured polyhedral grid was used with extruded regions upstream and downstream of the orifice region to help simulation converge. There were three different regions (Fig. 2) with Region 1 around orifice having finer mesh of around 130000 cells and upstream and downstream regions (Region 2 and Region 3) having coarser mesh with around 10000 and 120000 cells respectively.

\subsection{Governing Equations}

The governing equations for flow through $\mathrm{SHO}$ and $\mathrm{MHO}$ are given by

Continuity equation:

$\frac{\partial \rho}{\partial t}+\nabla \cdot\left(\rho v_{i}\right)=0$

Momentum equation:

$\frac{\partial}{\partial t}\left(\rho v_{i}\right)+\nabla \cdot\left(\rho v_{i} v_{i}\right)=-\nabla p+\nabla \cdot \tau$

Standard $k-\varepsilon$ turbulence model was used in numerous orifice CFD studies (Erdal and Andersson, 1997; Shah et al., 2012; Singh and John Tharakan, 2015), hence authors chose this turbulence model for computations. Standard $k-\varepsilon$ turbulence model is given by: 


\begin{tabular}{|c|c|c|c|}
\hline Region 2 & Region 3 \\
\hline $12 \mathrm{D}$ & Region & \\
\hline & $44 \mathrm{D}$ \\
\hline
\end{tabular}

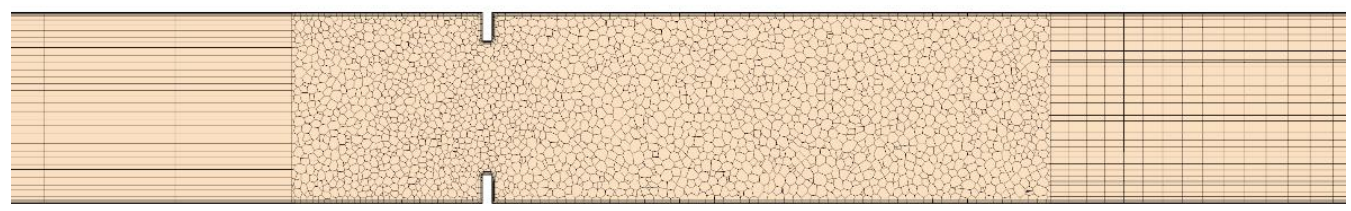

Fig. 2. Regions and Mesh.

$\frac{\partial}{\partial t}(\rho k)+\frac{\partial}{\partial x_{i}}\left(\rho k v_{i}\right)=\frac{\partial}{\partial x_{j}}\left[\left(\mu+\frac{\mu_{t}}{\sigma_{k}}\right) \frac{\partial k}{\partial x_{j}}\right]+G_{k}-\rho \varepsilon$

$\frac{\partial}{\partial t}(\rho \varepsilon)+\frac{\partial}{\partial x_{i}}\left(\rho \varepsilon v_{i}\right)=$

$=\frac{\partial}{\partial x_{j}}\left[\left(\mu+\frac{\mu_{t}}{\sigma_{\varepsilon}}\right) \frac{\partial \varepsilon}{\partial x_{j}}\right]+C_{1 \varepsilon} \frac{\varepsilon}{k} G_{k}-C_{2 \varepsilon} \rho \frac{\varepsilon^{2}}{k}$

Where $C_{1 \varepsilon}, C_{2 \varepsilon}, \sigma_{k}$ and $\sigma_{\varepsilon}$ are standard $k-\varepsilon$ turbulence model constants while $G_{k}$ is turbulent production and it is modelled as:

$$
G_{k}=\mu_{t} S^{2}-\frac{2}{3} \rho k \nabla \cdot v_{i}-\frac{2}{3} \mu_{t}\left(\nabla \cdot v_{i}\right)^{2}
$$

Turbulent viscosity is computed as:

$$
\mu_{t}=\rho C_{\mu} \frac{k^{2}}{\varepsilon}
$$

All governing equations were solved by segregated solver while all solutions were considered to be fully converged when each of the residuals was less than $10^{-6}$.

\subsection{Boundaries}

Air at $25^{\circ} \mathrm{C}$ was used as the working fluid. CFD simulations were carried out over a wide range of Reynolds numbers from 500 to 600000 while mass flow rate boundary condition was specified at the pipe inlet. Flow split outlet boundary represents the outlet of a duct and it was used to model flow exit with conservation of mass. No slip boundary condition was used for wall surfaces and high y+ wall treatment for near-wall modelling. This treatment assumes that the near-wall cell lies within the logarithmic region of the boundary layer (Siemens, 2019).

\subsection{Grid Sensitivity Analysis}

In order to find optimal mesh size for this study, grid sensitivity analysis was performed. Four different size meshes (Table 2) were studied where each had more cells than the previous. Same conditions $\left(Q=166.84 \quad \mathrm{~m}^{3} / \mathrm{h}, \quad p=399 \quad \mathrm{kPa}\right.$ and $t=300.18 \mathrm{~K}$ ) were used to simulate flow through the orifice for all meshes.

Table 2 Mesh sizes for grid analysis

\begin{tabular}{|c|c|c|c|}
\hline Mesh & Region 1 & Region 2 & Region 3 \\
\hline Mesh 1 & 17478 & 3330 & 37800 \\
\hline Mesh 2 & 29375 & 3240 & 40800 \\
\hline Mesh 3 & 129389 & 9680 & 118680 \\
\hline Mesh 4 & 298993 & 15340 & 182040 \\
\hline
\end{tabular}

Figure 3 shows pressure recovery variation for four types of chosen meshes. Two coarser meshes (Mesh 1 and Mesh 2) have significant change in pressure recovery pattern while finer meshes (Mesh 3 and Mesh 4) have no significant change in pressure recovery pattern.

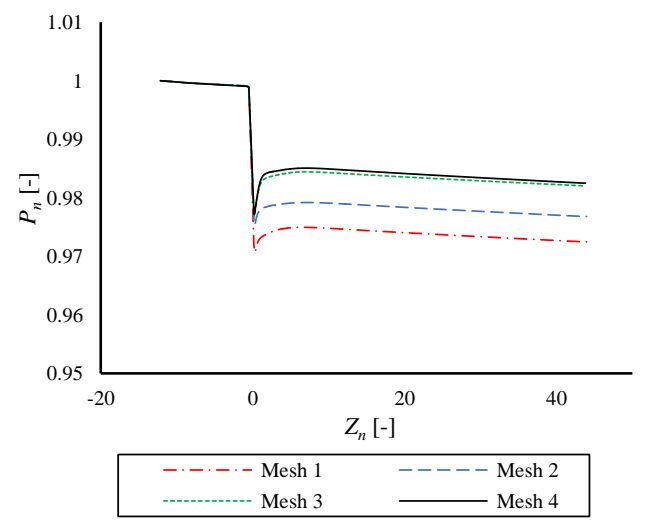

Fig. 3. Pressure profiles for different mesh size.

Convergence was assessed by monitoring pressure drop results and comparing them with experimental pressure drop result (Fig. 4). The difference between Mesh 3 and Mesh 4 is $1.01 \%$ for the pressure drop while the difference between Mesh 3 and experimental results is $0.88 \%$ compared to Mesh 4 where difference between simulation and experimental results is $0.12 \%$. Hence, Mesh 3 was chosen for further simulations. 


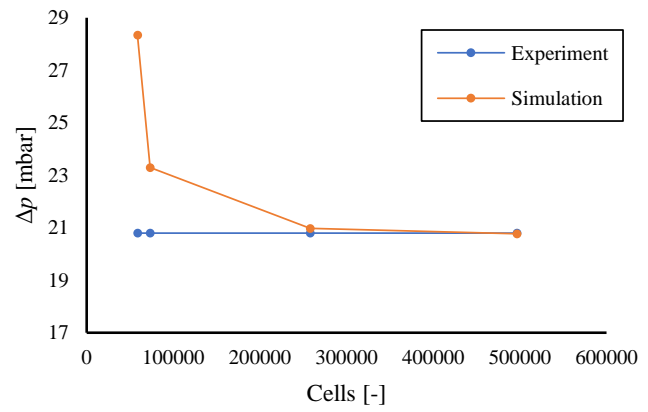

Fig. 4. Pressure drop comparison for different Meshes.

\subsection{CFD Model Validation}

CFD model was validated with experimental data of SHO and MHO flow meters (Đurđević et al., 2019). Details of test facility with straight sections of $12 \mathrm{D}$ upstream and $4 D$ downstream of the orifice are shown in Fig. 5. Elster quantometers type QAe250 and type QAe650 were used for air flowrate measurement with former having a measuring range (20-400) $\mathrm{m}^{3} / \mathrm{h}$ and latter $(50-1000) \mathrm{m}^{3} / \mathrm{h}$, with an accuracy of $+/-1.5 \%$ over a scale of $20 \%$ to $100 \%$ of $Q_{\max }$ while accuracy of $+/-3 \%$ was over a scale of $10 \%$ to $20 \%$ of $Q_{\max }$. Upstream pressure was measured with Yokogawa's absolute pressure transmitter model EJA510A with measuring range $(0-10) \mathrm{MPa}$ and accuracy of $+/-0.2 \%$. Pressure drop over orifice plates was measured with Yokogawa's differential pressure transmitter model EJA110A with measuring range $(0-50) \mathrm{kPa}$ and accuracy of $+/-0.065 \%$.

Figure 6 shows comparison of simulation and experimental results for singular pressure loss coefficient of SHO and MHO1 for $\beta=0.7$. Good agreement between the two has been achieved with maximum deviation being within $14 \%$ for $\mathrm{SHO}$ and $7 \%$ for MHO1. These results are validating adopted CFD model. Therefore, this model can be used for further computations of SHO and MHO with different $\beta$ ratios.

\section{RESULTS AND DISCUSSIONS}

Numerical procedure described in section 3 was used to simulate air flow through SHO and MHO. Figure 7 shows predicted velocity scalar fields (Re 70000) for SHO and $\mathrm{MHO} 2$ for $\beta=0.7$. Both SHO and MHO have equal open area, but MHO have more evenly spread area comparing to SHO where there is only one central opening (Fig. 1 and Fig. 7). With fluid passing through the SHO, large sized eddies are generated downstream of the plate comparing to $\mathrm{MHO}$ where smaller sized eddies are generated downstream.

Both SHO and MHO2 have equal upstream velocities close to the pipe wall, as it is shown in cross-sectional velocity distributions upstream of the orifice in Fig. 8 a) and 9 a). Figures 8 b) and 9 b) are showing cross-sectional velocities downstream of the orifice, where velocities close to the pipe wall for both $\mathrm{SHO}$ and $\mathrm{MHO} 2$ can be seen. From these figures, it can be seen that $\mathrm{MHO} 2$ has lower velocity close to the pipe wall compared to SHO. These trends are same for all simulated orifices, so for the corresponding SHO, MHO will have higher pressure downstream which is leading to lower pressure drop for same flow rate in favour of MHO.

Figure 9 b) shows cross-sectional pressure distribution downstream of the orifice for $\mathrm{MHO} 2$ where pressure is not evenly distributed especially close to the pipe wall. This can prove to be important when placing $\mathrm{MHO}$ in the pipe especially if pressure drop is measured $1 D$ upstream and $1 / 2 D$ downstream of the orifice. Orientation of MHO smaller openings and position of pressure taps should be taken into account, as this can lead to inadequate pressure drop measurements and indirectly incorrect flow rate. Although downstream pressure differences close to the pipe wall are not that great, they should be taken in consideration.

\subsection{Singular Pressure Loss Coefficient}

Variations in singular pressure loss coefficient of SHO and MHO plates are presented in Fig. 10. Four SHO (for $\beta$ : $0.5,0.55,0.6$ and 0.7) have pressure loss coefficient values of $2.304,2.182$, 2.077 and 1.732, respectively. Comparing simulation results it is evident that $\mathrm{MHO}$ have lower singular pressure loss coefficient than SHO. Greatest improvement in singular pressure loss coefficient can be seen for orifice plate $\mathrm{MHO} 3$ with $\beta$ ratios 0.5 and 0.55 and orifice plate $\mathrm{MHO} 2$ with $\beta$ ratios 0.6 and 0.7. Comparing to SHO of corresponding $\beta$ ratios, these MHO have a decrease of $30.81 \%, 31.46 \%, 32.57 \%$ and $32.39 \%$ in singular pressure loss coefficient for $\beta$ ratios of 0.5 , $0.55,0.6$ and 0.7 , respectively. Hole distribution is not as significantly as $\beta$ ratio affecting singular pressure loss coefficient where decreases of the coefficient influenced by hole distribution is by $10.00 \%, 10.68 \%, 14.45 \%$ and $4.86 \%$ for $\mathrm{MHO} 3$ with $\beta$ ratios 0.5 and 0.55 and $\mathrm{MHO} 2$ with $\beta$ ratios 0.6 and 0.7 respectively. This leads to a conclusion that orifice plates MHO3 with $\beta$ ratios 0.5 and 0.55 and orifice plates $\mathrm{MHO} 2$ with $\beta$ ratios 0.6 and 0.7 have optimal homocentric circle diameter in terms of singular pressure loss coefficient.

In Fig. 11 singular pressure loss coefficents are plotted for all simulated orifice plates at different Reynolds numbers making it possible to estimate which orifices have equivalent singular pressure loss coefficients and indirectly discharge coefficients. It can be seen that with $\beta$ ratio increase, orifices sensitivity to Reynolds numbers is decreasing while $\mathrm{MHO}$ are less sensitive to Reynolds number change than SHO. MHO singular pressure loss coefficient is not as influenced by Reynolds number change like SHO, making in this way MHO more applicable over wide range of Reynolds munbers. 
M. Đurđević et al. / JAFM, Vol. 14, No. 1, pp. 215-226, 2021.
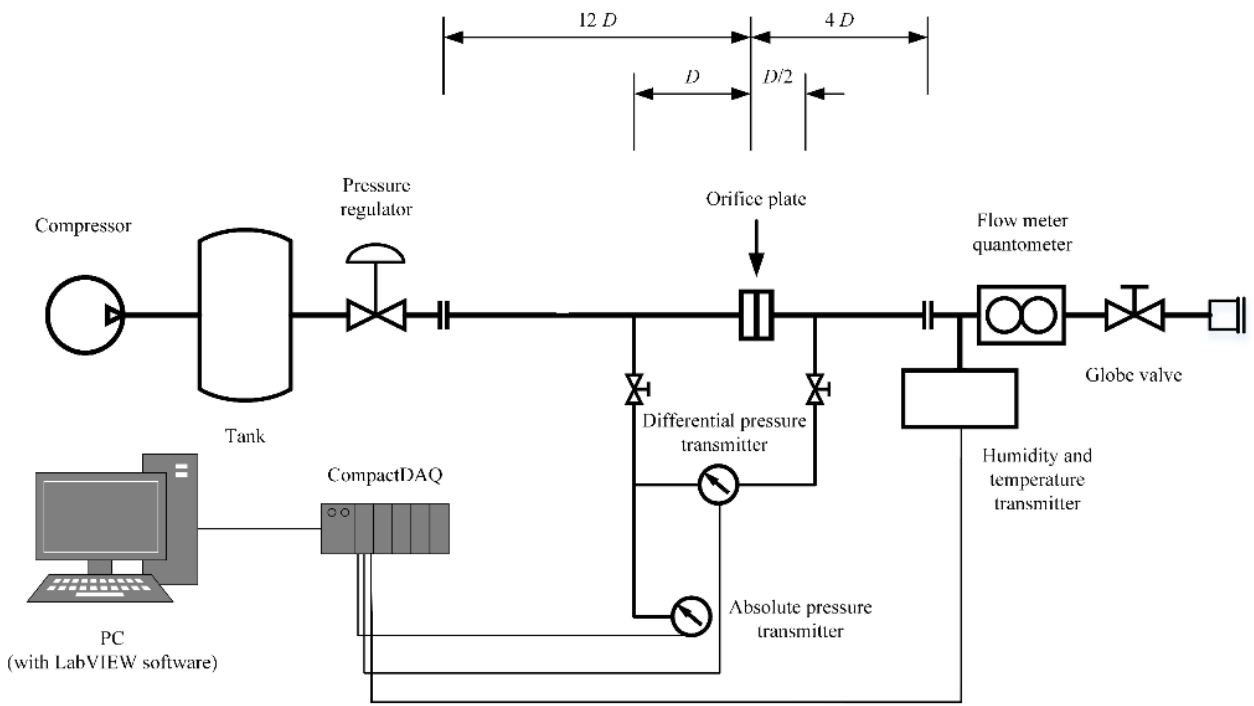

Fig 5. Test facility.

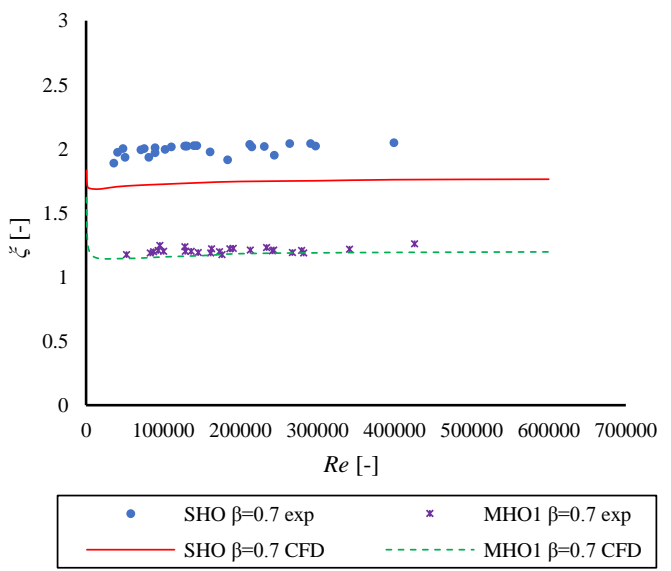

Fig. 6. Comparison of experimental and simulated results for $\mathrm{SHO}$ and MHO1 $\beta=0.7$.

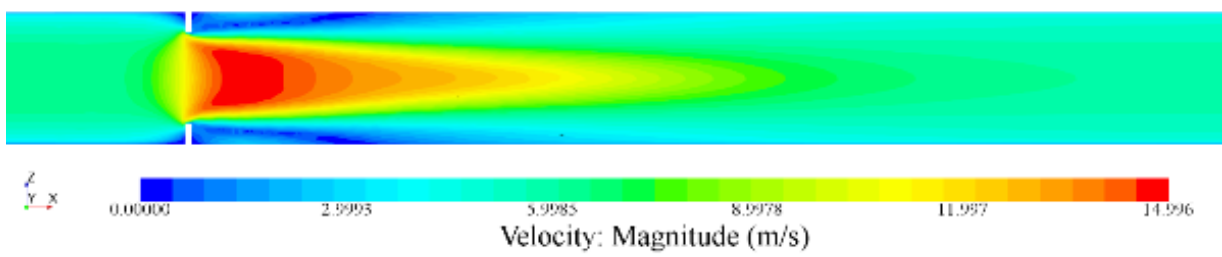

a) $\mathrm{SHO} \beta=0.7$

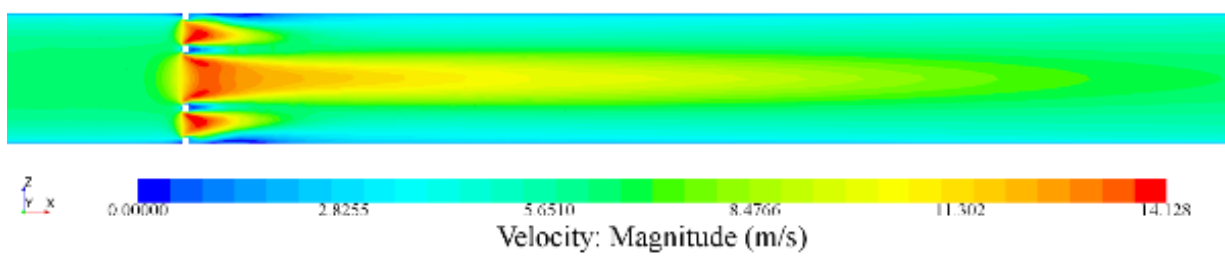

b) $\mathrm{MHO} 2 \beta=0.7$

Fig. 7. Downstream velocity scalar fields for $\mathrm{SHO}$ and $\mathrm{MHO} 2 \beta=0.7$ at $\operatorname{Re} 70000$. 

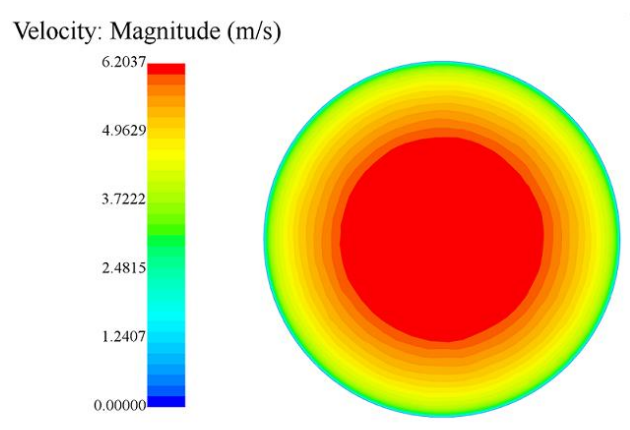

Velocity: Magnitude $(\mathrm{m} / \mathrm{s})$
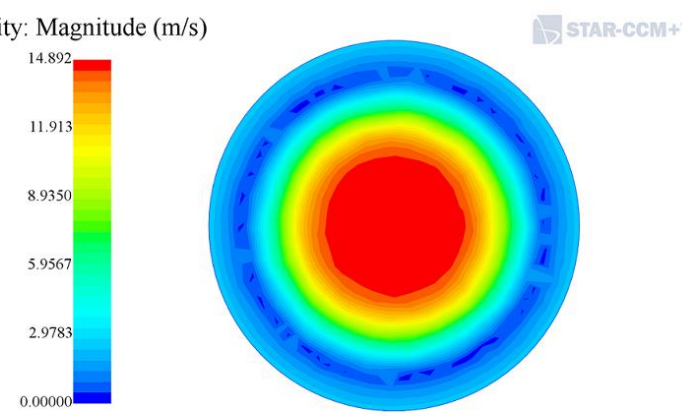

Pressure $(\mathrm{Pa})$
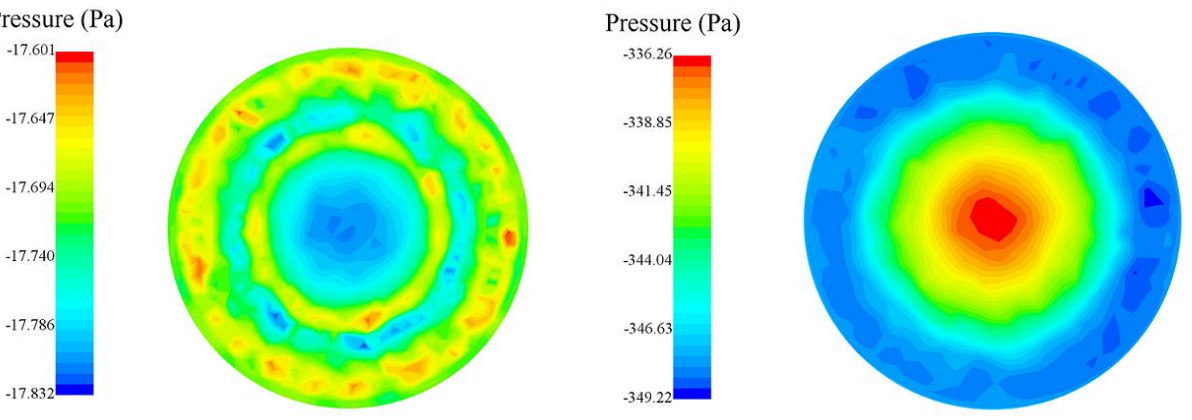

a) $1 D$ upstream

b) $1 / 2 D$ downstream

Fig. 8. Cross-sectional velocity and pressure distribution for SHO $\beta=0.7$ at $\operatorname{Re} 70000$.
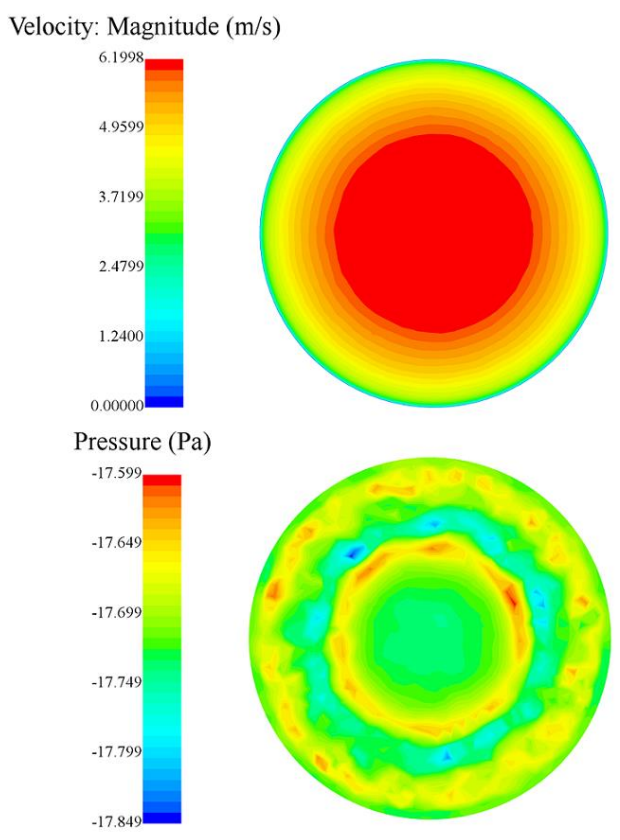

a) $1 D$ upstream

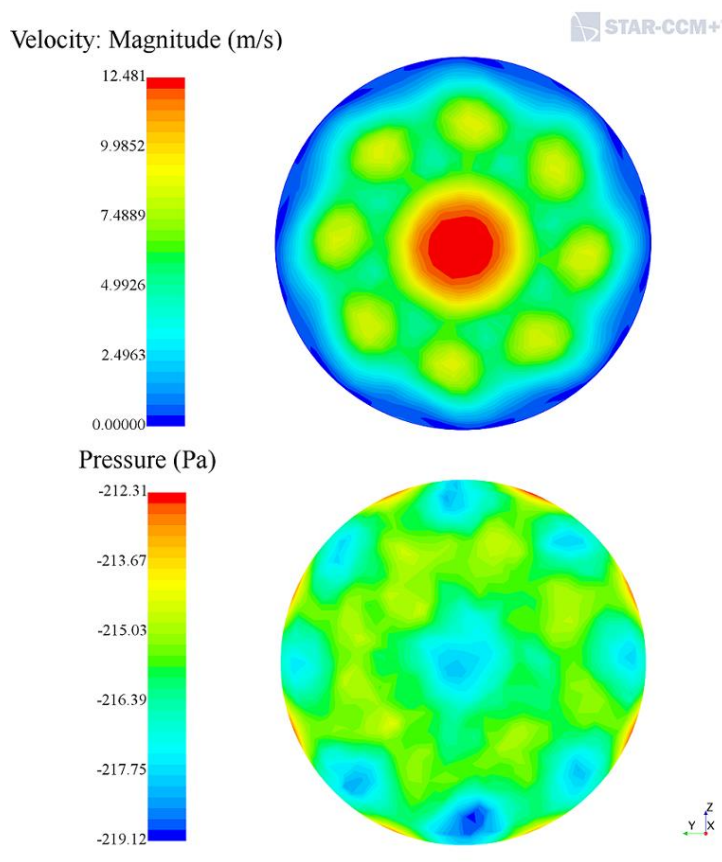

b) $1 / 2 D$ downstream

Fig. 9. Cross-sectional velocity and pressure distribution for $\mathrm{MHO} 2 \beta=0.7$ at $\operatorname{Re} 70000$.

Knowing that expansion factor $\varepsilon$ is very close to 1 , discharge coefficient $C_{d}$ can be determined using Eq. (5). It can be seen that with decrease of singular pressure loss coefficeint $\xi$, discharge coefficeint $C_{d}$ increases, therefore $\mathrm{MHO}$ have higher (better) discharge coefficient comparing to SHO.

\subsection{Pressure Recovery}

For all simulated orifices over the whole range of Reynolds numbers, MHO had faster pressure 


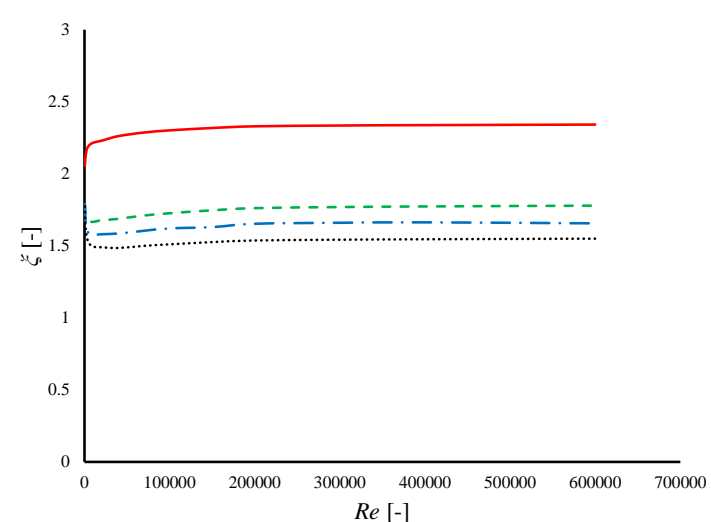

a) $\beta=0.5$

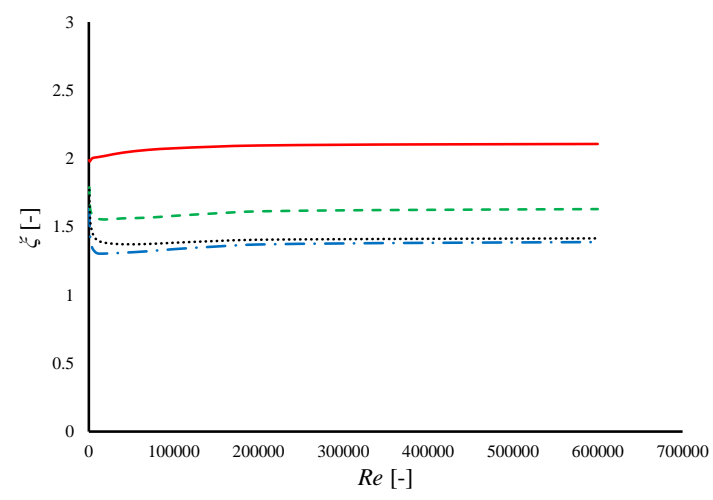

c) $\beta=0.6$

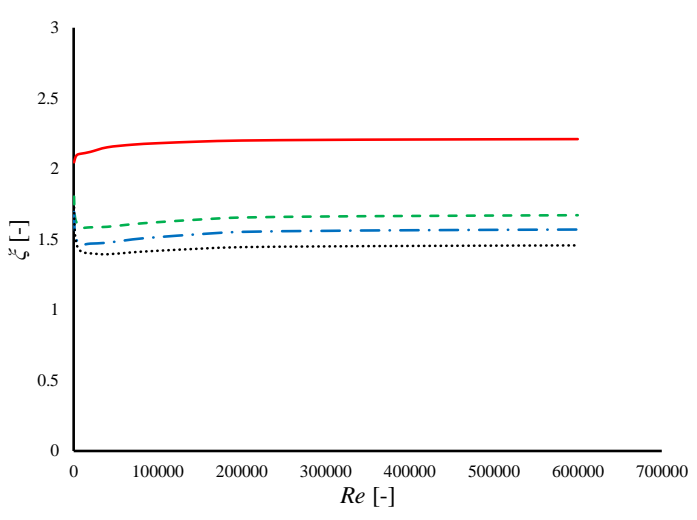

b) $\beta=0.55$

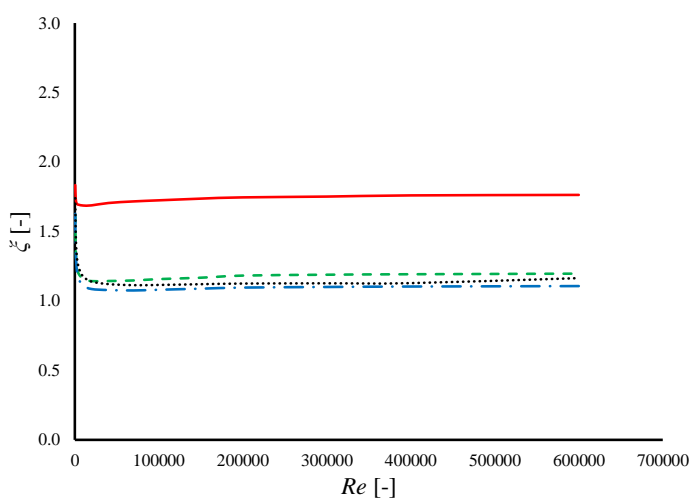

d) $\beta=0.7$

\begin{tabular}{|c|c|c|c|}
\hline$\longrightarrow \mathrm{SHO}$ & $----\mathrm{MHO} 1$ & $-\cdots-\mathrm{MHO} 2$ & ............... MHO3 \\
\hline
\end{tabular}

Fig. 10. Singular pressure loss coefficient for SHO and MHO.

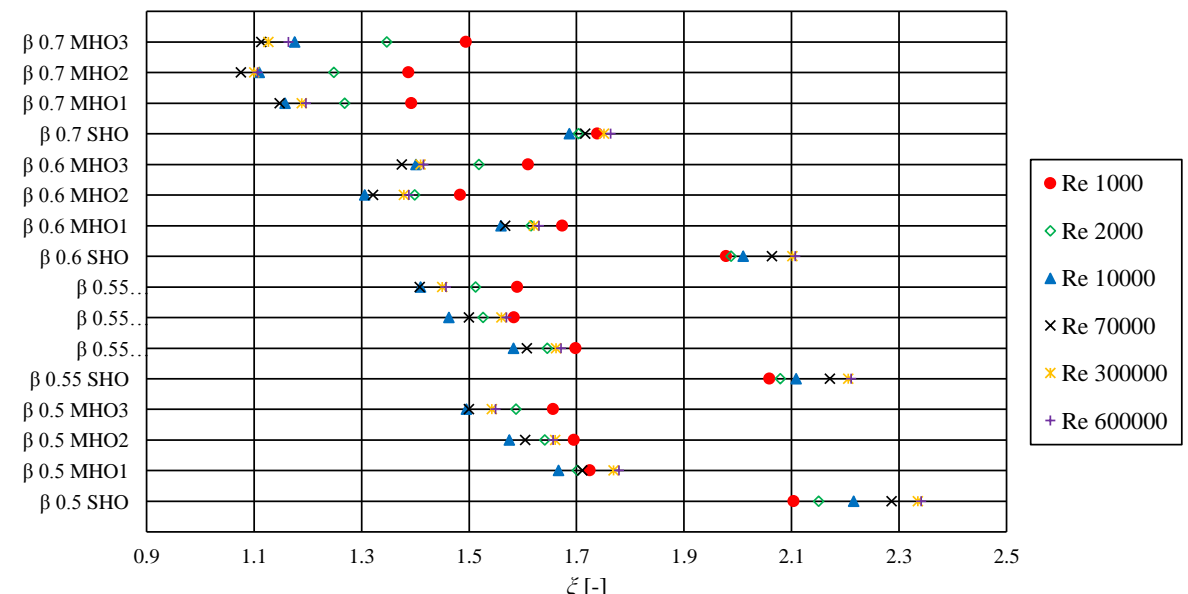

Fig. 11. Singular pressure loss coefficients at different Reynolds numbers.

recovery compared to SHO. Figure 12 shows pressure recovery pattern for $\mathrm{SHO}$ and $\mathrm{MHO}$ with optimal homocentric circle diameter for all $\beta$ ratios at different Reynolds numbers. At low Reynolds numbers (Re 1000) SHO have slightly greater pressure recovery compared to $\mathrm{MHO}$. With an increase of Reynolds number to 3000 , MHO with $\beta$ ratios of $0.5,0.55,0.6$ have slightly greater pressure recovery then corresponding $\mathrm{SHO}$, while $\mathrm{MHO}$ with $\beta=0.7$ still has lower pressure recovery 


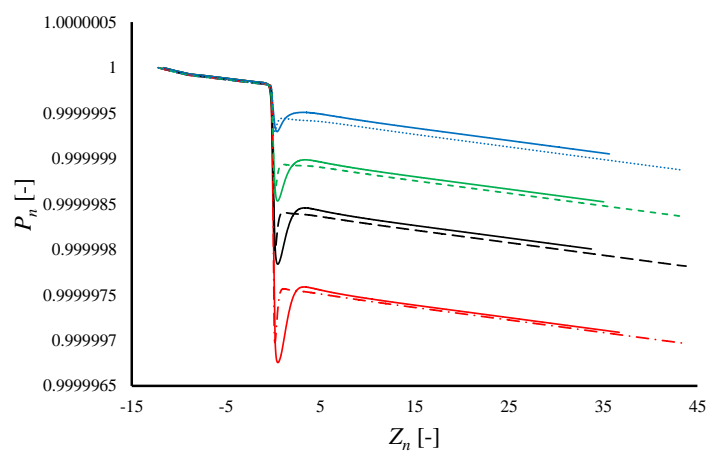

a) $\quad \operatorname{Re} 1000$

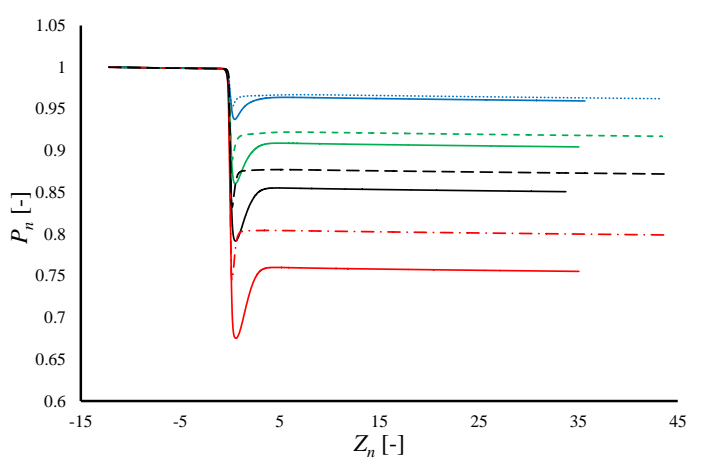

c) $\operatorname{Re} 300000$

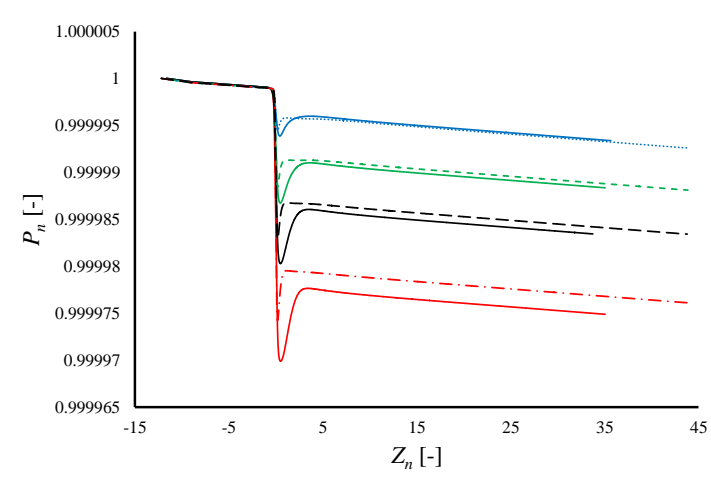

b) $\operatorname{Re} 3000$

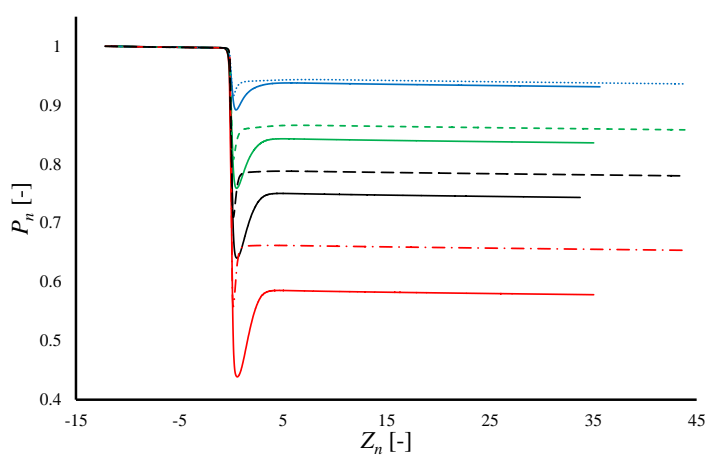

d) $\operatorname{Re} 600000$

\begin{tabular}{|c|c|c|c|}
\hline$\longrightarrow$ SHO $\beta=0.5$ & $\longrightarrow$ SHO $\beta=0.55$ & $\longrightarrow$ SHO $\beta=0.6$ & $\longrightarrow$ SHO $\beta=0.7$ \\
\hline$-\cdots$ МHO3 $\beta=0.5$ & - $\mathrm{MHO} 3 \beta=0.55$ & $---\mathrm{MHO} 2 \beta=0.6$ & $\cdots \cdots \cdots \cdot$ MHO2 $\beta=0.7$ \\
\hline
\end{tabular}

Fig. 12. Pressure recovery for SHO and MHO with optimal homocentric circle diameter.

compared to corresponding SHO. At lower Reynolds numbers (1000 and 3000) pressure recovery both for SHO and MHO is significant as almost all lost pressure is recovered, but pressure recovery rate is insignificant. Pressure recovery at higher Reynolds numbers (300000 and 600000) is greater for $\mathrm{MHO}$ compared to $\mathrm{SHO}$ for all simulated orifices.

Table 3 shows pressure recovery rates for higher Reynolds numbers and their improvements. It can be seen that with an increase of Reynolds number pressure recovery is decreasing. Greatest improvement of $7.7 \%$ was found for $\mathrm{MHO} 3$ with $\beta=0.5$ at Reynolds number of 600000 while the least improvement of $0.2 \%$ for $\mathrm{MHO} 2$ with $\beta$ ratio 0.7 at Reynolds number of 200000.

\subsection{Axial Velocity and Vena-Contracta}

The centreline axial velocity profiles for SHO and MHO are given in Fig. 13. In inlet section of the pipe, flow is uniform i.e. centreline axial velocity is unchanged. As approaching the orifice opening centreline axial velocity is increasing both for SHO and MHO flow meters. Maximum centreline axial velocity can be observed downstream of the orifice while the pressure has it lowest value at this point.
This point of the flow stream where the diameter of the stream is the least and fluid velocity at its maximum is called vena-contracta. Beyond this point velocity is just decreasing to the approximate value it had at pipe inlet section just before the orifice, which was the case for all simulated orifices.

Table 3 Pressure recovery for higher Reynolds numbers

\begin{tabular}{|c|c|c|c|c|}
\hline \multirow{3}{*}{$\begin{array}{c}\beta \\
{[-]}\end{array}$} & Orifice & \multicolumn{3}{|c|}{$R e$} \\
\cline { 3 - 5 } & & 200000 & 300000 & 600000 \\
\hline \multirow{3}{*}{0.5} & SHO & $89.4 \%$ & $76.1 \%$ & $58.5 \%$ \\
\cline { 2 - 5 } & MHO3 & $91.3 \%$ & $80.4 \%$ & $66.2 \%$ \\
\cline { 2 - 5 } & Improvement & $1.9 \%$ & $4.3 \%$ & $7.7 \%$ \\
\hline \multirow{3}{*}{0.55} & SHO & $93.6 \%$ & $85.5 \%$ & $75.1 \%$ \\
\cline { 2 - 5 } & MHO3 & $94.6 \%$ & $87.7 \%$ & $78.8 \%$ \\
\cline { 2 - 5 } & Improvement & $1.0 \%$ & $2.2 \%$ & $3.7 \%$ \\
\hline \multirow{3}{*}{0.6} & SHO & $95.9 \%$ & $90.9 \%$ & $84.3 \%$ \\
\cline { 2 - 5 } & MHO2 & $96.5 \%$ & $92.2 \%$ & $86.6 \%$ \\
\cline { 2 - 5 } & Improvement & $0.7 \%$ & $1.3 \%$ & $2.3 \%$ \\
\hline \multirow{3}{*}{0.7} & SHO & $98.3 \%$ & $96.4 \%$ & $93.8 \%$ \\
\cline { 2 - 5 } & MHO2 & $98.5 \%$ & $96.7 \%$ & $94.3 \%$ \\
\cline { 2 - 5 } & Improvement & $0.2 \%$ & $0.3 \%$ & $0.5 \%$ \\
\hline
\end{tabular}




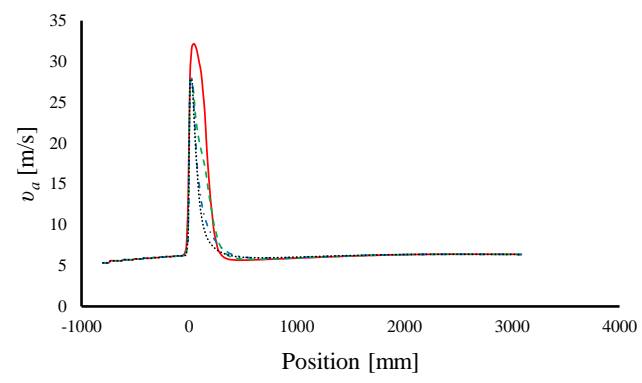

a) $\quad \beta=0.5$

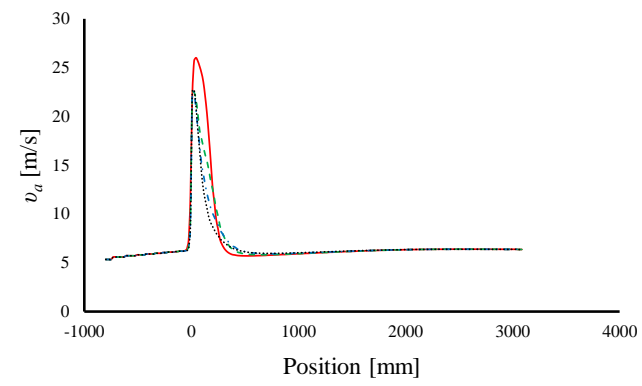

b) $\beta=0.55$

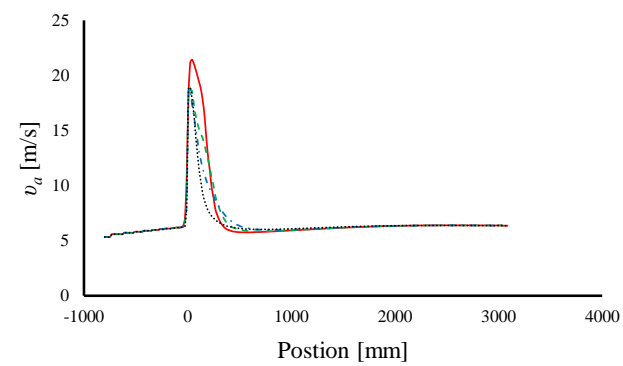

c) $\quad \beta=0.6$

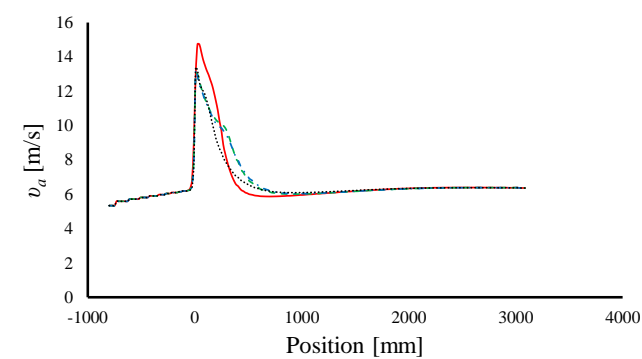

d) $\quad \beta=0.7$

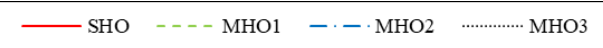

Fig. 13. Centreline axial velocity for SHO and MHO at Re 70000.

All simulated MHO flow meters had lower centreline axial velocity compared to corresponding SHO. Decrease in centreline axial velocity was $16.33 \%, 15.75 \%, 14.38 \%$ and $12.9 \%$ for orifices with $\beta$ ratios $0.5,0.55,0.6$ and 0.7 , respectively. Hole distribution influence was insignificant to maximum centreline velocity.

From Fig. 13. it can be seen that, vena-contracta position is different for SHO and MHO flow meters. For all simulated orifices, MHO venacontracta position was closer to orifice plate compared to SHO. This could lead to a conclusion that for MHO flow meters moving downstream pressure tap location closer to the orifice plate (vena-contacta) greater pressure drop could be recorded. In this way it would be possible to use pressure measuring instruments of lower quality than if downstream pressure tap location was on $D / 2$ distance from the orifice plate.

\section{CONCLUSION}

In this study $\mathrm{SHO}$ and $\mathrm{MHO}$ with four different $\beta$ ratios $(0.5,0.55,0.6$ and 0.7$)$ were numerically studied over a wide range of Reynolds numbers with air as a working fluid. Numerical model was compared to experimental results and good agreement was achieved.

SHO and MHO singular pressure loss coefficients were studied by varying structural parameters like hole size ( $\beta$ ratio) and hole distribution. Simulation results showed a significant decrease of MHO singular pressure loss coefficient compared to SHO by $30.81 \%, 31.46 \%, 32.57 \%$ and $32.39 \%$ for $\beta$ ratios $0.5,0.55,0.6$ and 0.7 respectively. Not so significant influence was recorded on singular pressure loss coefficient by MHO hole distribution. Beside singular pressure loss coefficient, pressure recovery for both the SHO and MHO was reported. Comparing to SHO faster pressure recovery was recorded with all simulated MHO. MHO had greater pressure recovery compared to corresponding $\mathrm{SHO}$ at $R e>3000$. This can be attributed to MHO geometry, as they have more evenly spread open area compared to SHO and in that way causing less of an obstruction in fluid flow. Greatest pressure recovery was recorded for MHO with $\beta=0.5$ as well as pressure recovery increase with $\beta$ ratio increase. Lower velocities downstream of the MHO are leading to pressure drop decrease thus requiring better pressure measuring devices compared to SHO for the same flow rates. Centreline axial velocity analyses showed that MHO flow meters have lower axial velocity compared to $\mathrm{SHO}$ as well as different vena-contracta position.

Orifice flow meters are still very popular measuring flow instruments due to its affordability, ease of use, reliability, simplicity, maintenance etc., but they also have disadvantages like low pressure recovery. Presented results showed advantages of MHO compared to SHO in singular pressure loss coefficient and pressure recovery and in this way put MHO one step closer of becoming a drop-in replacement for the SHO, but further research is needed.

\section{ACKNOWLEDGMENTS}

The authors gratefully acknowledge all support from GasTeh d.o.o. Inđija that allowed use of their test facility as well as manufacturing all necessary orifices and parts for the study. This paper is a result of research within the project TR31058, 2011 - 2019, financially supported by the Ministry of 
Education, Science and Technological Development, Republic of Serbia.

\section{REFERENCES}

Arun, N., S. Malavarayan and M. Kaushik (2010). CFD Analysis on Discharge Co- Efficient During Non- Newtonian Flows Through Orifice Meter, International Journal of Engineering Science and Technology 2(7), 3151-3164.

Barki, M. and T. Ganesha (2014). CFD Analysis and Comparison of Fluid Flow Through A Single Hole And Multi Hole Orifice Plate, International Journal of Research in Advent Technology 2(8), 6-15.

Dong, J., C. Jing, Y. Peng, Y. Liu, H. Ren and X. Liu (2018). Study on the measurement accuracy of an improved cemented carbide orifice flowmeter in natural gas pipeline, Flow Measurement and Instrumentation 59, 52-62.

Drainy, Y., K. Saqr, H. Aly, M. Nazri and M. Jaafar (2009). CFD analysis of incompressible turbulent swirling flow through zanker plate, Engineering Applications of Computational Fluid Mechanics 3(4), 562-572.

Đurđević, M., M. Bukurov, S. Tašin and S. Bikić (2019) Experimental research of single-hole and multi-hole orifice gas flow meters, Flow measurement and instumentation. 70, 101650.

Durst, F. and A. B. Wang (1989). Experimental and numerical investigations of the axisymmetric, turbulent pipe flow over a wall-mounted thin obstacle, in Symposium on Turbulent Shear Flows, 7th, Stanford, CA, Aug. 21-23, 1989, Proceedings. Volume 1 (A90-35176 15-34). University Park, PA, Pennsylvania State University 1989, 10.4.1-10.4.6.

Eiamsa-Ard, S., A. Ridluan, P. Somravysin and P. Promvonge (2008). Numerical investigation of turbulent flow through a circular orifice, KMITL Science and Technology Journal 8(1), 43-50.

Ellman, A. and R. Piche' (1999). A Two Regime Orifice Flow Formula for Numerical Simulation, Journal of Dynamic Systems, Measurement 121(4), 721-724.

Elsaey, A., A. Abou El-Azm Aly and M. Fouad (2014). CFD simulation of fractal-shaped orifices for flow measurement improvement, Flow Measurement and Instrumentation 36, 14-23.

Erdal, A. and H. Andersson (1997) 'Numerical aspects of flow computation through orifices, Flow Measurement and Instrumentation 8(1), 27-37.

Gan, G. and S. B. Riffat (1997). Pressure loss characteristics of orifice and perforated plates, Experimental Thermal and Fluid Science
14(2), 160-165.

Hollingshead, C. L., M. C. Johnson, S. L. Barfuss and R. E. Spall (2011). Discharge coefficient performance of Venturi, standard concentric orifice plate, V-cone and wedge flow meters at low Reynolds numbers, Journal of Petroleum Science and Engineering 78(3-4), 559-566.

Morrison, G. L., R. E. Deotte Jr, G. H. Nail and D. L. Panak (1993). Mean velocity and turbulence fields inside a $\beta=0.50$ orifice flowmeter, AIChE Journal 39(5), 745-756.

Morrison, G. L., K. R. Hall, J. C. Holste, M. L. Macek, L. M. Ihfe, R. E. Deotte Jr and D. P. Terracina (1994). Comparison of orifice and slotted plate flowmeters, Flow Measurement and Instrumentation 5(2), 71-77.

Nail, G. H. (1991) A study of 3-Dimensional flow through orifice meters. Ph. D. thesis, Texas A\&M University, USA.

Ntamba Ntamba, B. and V. Fester (2012). Pressure Losses and Limiting Reynolds Numbers for Non-Newtonian Fluids in Short Square-Edged Orifice Plates. Journal of Fluids Engineering 134(9).

Oliveira, N. M. B., L. G. M. Vieira and J. J. R. Damasceno (2010). Numerical Methodology for Orifice Meter Calibration, Materials Science Forum, 660-661(November 2015), 531-536.

Reader-Harris, M. (2015) Orifice Plates and Venturi Tubes. Springer International Publishing, Switzerland.

Reader-Harris, M., N. Barton and D. Hodges (2012). The effect of contaminated orifice plates on the discharge coefficient, Flow Measurement and Instrumentation 25, 2-7.

Siemens (2019).User Guide STAR CCM+.

Shaaban, S. (2014). Optimization of orifice meter's energy consumption, Chemical Engineering Research and Design 92(6), 1005-1015.

Shah, M., J. Joshi, A. Kalsi, C. Prasad and D. Shukla (2012). Analysis of flow through an orifice meter: CFD simulation, Chemical Engineering Science 71, 300-309.

Singh, R. K., S. N. Singh and V. Seshadri (2010). CFD prediction of the effects of the upstream elbow fittings on the performance of cone flowmeters, Flow Measurement and Instrumentation 21(2), 88-97.

Singh, V. K. and T. John Tharakan (2015) 'Numerical simulations for multi-hole orifice flow meter, Flow Measurement and Instrumentation 45, 375-383.

Teyssandier, R. G. and Z. D. Husain (1987). Experimental Investigation of an Orifice Meter Pressure Gradient. Journal of Fluids Engineering 109(2), 144-148. 